No absolute convergence proof was found for the iteration process itself; on the other hand, no convergence instabilities were experienced in the actual computation for the range of parameters that was tabulated.

Acknowledgement. The author wishes to express his indebtedness to Professor A. A. Oliner for his interest and help in connection with this paper, and to Dr. Gertrude Blanch, Aeronautical Research Laboratory, for providing valuable references and information. Special thanks are due to John Segal for his patience and ingenuity in carrying out the programming and obtaining the numerical results.

Microwave Research Institute

Polytechnic Institute of Brooklyn

Brooklyn, New York

1. N. W. Mclachlan, Theory and Application of Mathieu Functions, University Press, Oxford, 1951.

2. J. MeIXner \& F. W. Schärke, Mathieusche Funktionen und Sphäroidfunktionen, Springer-Verlag, Berlin, 1954 .

3. Nat. BUR. STandard, Tables Relating to Mathieu Functions, Columbia University Press, New York, 1951.

4. J. C. WILTSE \& M. J. KING, Values of Mathieu Functions, Johns Hopkins Radiation Laboratory, Technical Report No. AF-53, 1958.

5. J. G. BRaINERd \& C. N. Weygand, "Solutions of Mathieu's equations," Phil. Mag., v. 30,1940, p. $458-477$.

6. H. J. Gray, R. Merwin \& J. G. Brainerd, "Solution of the Mathieu equation," Amer. Inst. Electr. Engrs., v. 67, 1948, p. 429-441.

7. S. J. ZAROODNY, An Elementary Review of the Mathieu-Hill Equation of Real Variables Based on Numerical Solutions, Ballistic Research Laboratories, Aberdeen Proving Ground Maryland, Memo. Rep. No. 878, April 1955.

\title{
An Algorithm for Solving Certain Polynomial Equations with Coefficient Parameters
}

\author{
By Robert D. Stalley
}

1. Introduction and General Method. We describe a storage-saving procedure that can be used in real time simulation or other situations in which the roots of an equation of the form

$$
\begin{aligned}
\sum_{j=0}^{n} F_{j}\left(x_{1}, x_{2}, \cdots, x_{m}\right) y^{e_{j}}=0, \quad m \geqq 2, \quad 0 \leqq e_{0} \leqq e_{1} \leqq & \cdots \leqq e_{n}, \\
& e_{j} \text { integral }(j=0,1, \cdots, n),
\end{aligned}
$$

must be obtained within severe time limits, i.e., from storage, for values of the coefficient parameter $m$-tuple $\left(x_{1}, x_{2}, \cdots, x_{m}\right)$ from some given set. The exponents $e_{j}$ are defined so as to be monotonically increasing rather than strictly increasing for later notational convenience.

Suppose there exist relations

$$
u_{i}=\varphi_{i}\left(x_{1}, x_{2}, \cdots, x_{m}\right), \quad(i=\mu, \mu+1, \cdots, m), 2 \leqq \mu \leqq m,
$$

Received February 21, 1961. 
which give the reduction

$$
\begin{aligned}
& F_{j}\left(x_{1}, x_{2}, \cdots, x_{m}\right) \\
& \quad=g\left(x_{1}, x_{2}, \cdots, x_{m}\right) f_{j}\left(u_{\mu}, u_{\mu+1}, \cdots, u_{m}\right) h^{e_{j}}\left(x_{1}, x_{2}, \cdots, x_{m}\right), \\
& \quad(j=0,1, \cdots, n) .
\end{aligned}
$$

Then, if we substitute from (3) into (1), setting

$$
y h\left(x_{1}, x_{2}, \cdots, x_{m}\right)=v \text {, }
$$

and divide out $g\left(x_{1}, x_{2}, \cdots, x_{m}\right)$, we obtain

$$
\sum_{j=0}^{n} f_{j}\left(u_{\mu}, u_{\mu+1}, \cdots, u_{m}\right) v^{e_{j}}=0
$$

Now, if we store the roots of equation (5) for all values of the $(m-\mu+1)$-tuple $\left(u_{\mu}, u_{\mu+1}, \cdots, u_{m}\right)$ within its range, we can find the roots of equation (1) for a given value of the $m$-tuple $\left(x_{1}, x_{2}, \cdots, x_{m}\right)$ within its domain of substitution by computing $\left(u_{\mu}, u_{\mu+1}, \cdots, u_{m}\right)$ from (2), obtaining from storage the roots of (5), and computing the roots of (1) from (4). Obvious separate considerations must apply if the value of $h\left(x_{1}, x_{2}, \cdots, x_{m}\right)$ or $g\left(x_{1}, x_{2}, \cdots, x_{m}\right)$ is zero or if any of the above functions are undefined for the given value of $\left(x_{1}, x_{2}, \cdots, x_{m}\right)$.

Basically we have replaced $m$-parameter storage by $(m-\mu+1)$-parameter storage and a computation which is ordinarily relatively simple. Rather than storing solutions point-wise in $m_{c}$-dimensional space, we store them by $(\mu-1)$ dimensional manifolds, namely those given by equations (2) taken simultaneously. A side calculation, using equation (4), is needed to obtain the roots for a particular point of the manifold.

This reduction is especially interesting when $\mu=m$, for then we need only single parameter storage. Note that if $m=2$ we must have $\mu=m$.

We consider two equations for which this reduction can be made. In both equations $\mu=2$.

2. An Equation of Power-Type. Let equation (1) be

$$
\sum_{j=0}^{n} a_{j}\left(\prod_{i=1}^{m} x_{i}^{\alpha_{i}}\right) y^{e_{j}}=0, \quad a_{j} \neq 0, \quad(j=0,1, \cdots, n),
$$

where

$$
\begin{array}{r}
\alpha_{1}+\sum_{i=2}^{m} r_{i} \alpha_{i}=k+r e_{j}, \quad\left(r_{2}, r_{3}, \cdots, r_{m}, r, k\right. \text { constant; } \\
\left.\alpha_{1}, \alpha_{2}, \cdots, \alpha_{m} \text { constant for each value of } j\right) .
\end{array}
$$

To reduce equation (6), we first use (7) to obtain

$$
\prod_{i=1}^{m} x_{i}^{\alpha_{i}}=x_{1}^{k} x_{1}^{r e_{j}} \prod_{i=2}^{m} x_{1}^{-r_{i} \alpha_{i}} \prod_{i=2}^{m} x_{i}^{\alpha_{i}}=x_{1}^{k}\left\{\prod_{i=2}^{m}\left(x_{1}^{-r_{i}} x_{i}\right)^{\alpha_{i}}\right\} x_{1}^{r e_{j}}
$$


Then, after dividing out $x_{1}{ }^{k}$, equation (6) becomes

$$
\sum_{j=0}^{n} a_{j}\left(\prod_{i=2}^{m} u_{i}^{\alpha_{i}}\right) v^{e_{j}}=0
$$

where

$$
u_{i}=x_{1}^{-r_{i}} x_{i}, \quad(i=2,3, \cdots, m),
$$

and

$$
v=x_{1}^{r} y
$$

This completes the reduction.

Homogeneous polynomial equations, with $y$ as one of the variables, are special cases where $r_{2}=r_{3}=\cdots=r_{m}=1, \quad r=-1$, and $\alpha_{i}$ is a non-negative integer, $(i=1,2, \cdots, m)$.

Now we look at the case $m=2$. Set $x_{1}=w, x_{2}=z, \alpha_{1}=\alpha, \alpha_{2}=\beta, r_{2}=s$, $r=t$, and $u_{2}=b$. The equation

$$
\sum_{j=0}^{n} a_{j} w^{\alpha} z^{\beta} y^{e_{j}}=0, \quad\left(\alpha+s \beta=k+t e_{j}\right),
$$

is reduced to the equation

$$
\sum_{j=0}^{n} a_{j} b^{\beta} v^{e_{j}}=0
$$

where

$$
b=w^{-8} z
$$

and

$$
v=w^{t} y .
$$

Thus, if the roots of (9) are stored for all of the values of $b$ in its range, we have the following algorithm for solving equation (8) for given values of $w$ and $z$ :

I. Compute $w^{s}$ and $w^{t}$;

II. Compute $b$ from (10);

III. Look up the roots of (9);

IV. Compute $y$ from (11).

The algorithm is especially simple if $s$ and $t$ are integers. We have combined storage lookup and computation to obtain an algorithm for solving (8) which involves little computation and only single parameter storage. The writer once needed a real time series solution of an equation of the form of (8) where $e_{n}=6, s=\frac{2}{3}$, $t=-\frac{1}{3}$, and where, of course, the remaining arbitrary constants were equal to specified values [1].

This equation of power type, equation (6) with condition (7), has many generalizations which are reducible in the same way. The functions $F_{j}$ may be quotients of polynomials whose terms are of the form of the coefficients of equation (6), where $e_{j}$ is constant for all terms of $F_{j}$, where $k$ and $r$ are constant for each polynomial, and where the values of $k$ and $r$ for any numerator minus the corresponding 
values of $k$ and $r$ for the corresponding denominator are constant. Moreover, the terms may contain other factors and the parameters may themselves be functions of other parameters.

3. An Equation of Exponential Type. Let equation (1) be

$$
\sum_{j=0}^{n} a_{j}\left(\prod_{i=1}^{m} \alpha_{i}^{x_{i}}\right) y^{e_{j}}=0, \quad a_{j} \neq 0, \quad(j=0,1, \cdots, n),
$$

where

$$
\begin{aligned}
& \alpha_{1} \prod_{i=2}^{m} \alpha_{i}^{r_{i}}=k c^{e_{j}}, \quad\left(r_{2}, r_{3}, \cdots, r_{m}, c, k\right. \text { constant; } \\
& \left.\quad \alpha_{1}, \alpha_{2}, \cdots, \alpha_{m} \text { constant for each value of } j\right), c \neq 0, k \neq 0 .
\end{aligned}
$$

To reduce equation (12), we first use (13) to obtain

$$
\prod_{i=1}^{m} \alpha_{i}^{x_{i}}=k^{x_{1}} c^{x_{1} e_{j}} \prod_{i=2}^{m} \alpha_{i}^{-r_{i} x_{1}} \prod_{i=2}^{m} \alpha_{i}^{x_{i}}=k^{x_{1}}\left(\prod_{i=2}^{m} \alpha_{i}^{-r_{i} x_{1}+x_{i}}\right) c^{x_{1} e_{j}}
$$

Then, after dividing out $k^{x_{1}}$, equation (12) becomes

$$
\sum_{j=1}^{n} a_{j}\left(\prod_{i=2}^{m} \alpha_{i}^{u_{i}}\right) v^{e_{i}}=0
$$

where

$$
u_{i}=x_{i}-r_{i} x_{1}, \quad(i=2,3, \cdots, m),
$$

and

$$
v=c^{x_{1}} y \text {. }
$$

This completes the reduction.

There are specializations and generalizations of this equation of exponential type analogous to those for the equation of power type.

4. Remarks. The restriction that $e_{j}$ be integral, $(j=0,1, \cdots, n)$, is not used and hence may be removed throughout this paper. With the customary interpretation of notation and statements, we may extend this paper to cover the trivial case $\mu=m+1$, and consequently the trivial case $m=1$.

An open problem is the enumeration of all examples, from various specified classes, for which the method of this paper is applicable. Another open problem, perhaps more interesting, is that of solving approximately polynomial equations with coefficient parameters by finding and solving approximating polynomial equations of a type for which the method of this paper is applicable. The generality of equation (6) with condition (7) and equation (12) with condition (13) make them promising approximating polynomial equations.

Oregon State Ǔniversity

Corvallis, Oregon

1. R. D. Stalley, Solution of a Polynomial Equation, Remington Rand, Inc., Engineering Research Associates Division, St. Paul, 1955, limited distribution. 